\title{
HIV patients dying on anti-tuberculosis treatment: are undiagnosed infections still a problem in French Guiana?
}

\author{
Mathieu Nacher ${ }^{1,2 *}$, Antoine Adenis ${ }^{1}$, Philippe Abboud ${ }^{3}$, Felix Djossou ${ }^{2,3}$, Magalie Demar ${ }^{4,5}$, Loïc Epelboin ${ }^{3}$ \\ and Pierre Couppié 2,6
}

\begin{abstract}
Objective: Despite scaling-up testing and antiretroviral treatment in Latin America, advanced HIV remains a significant public health problem. The objective of the present study was look for historical risk factors for death in French Guiana's HIV cohort taking into account the immunological status, the main opportunistic infections, and their treatment. A retrospective cohort study was conducted on data collected between 1992 and 2008 to identify factors associated with death in a cohort 2323 patients.

Results: There were 370 deaths for a total 9608 patient-years. Being on tuberculosis treatment was associated with a greater hazard of death. The diagnosis of confirmed tuberculosis, of histoplasmosis, of toxoplasmosis, and pneumocystosis were independently associated with death. Interactions terms between cotrimoxazole treatment and pneumocystosis, or between confirmed tuberculosis and tuberculosis treatment showed a protective treatment-effect. All patients having received anti-tuberculosis treatment $(n=347)$ did not have a final diagnosis of tuberculosis $(n=93)$. For histoplasmosis, 199 patients received antifungal treatment while 141 were diagnosed as having histoplasmosis. The number of patients on anti-tuberculosis drugs was far greater that the number of patients with confirmed tuberculosis, and these patients on treatment without confirmed tuberculosis had a twofold greater risk of dying.
\end{abstract}

Keywords: AIDS, Tuberculosis, Histoplasmosis, Death, Diagnosis, Latin America

\section{Introduction}

Disseminated histoplasmosis and tuberculosis are 2 very common opportunistic infections of patients with advanced HIV disease in Latin America. It is not easy to differentiate them because rapid diagnostic tests for histoplasmosis are lacking in most of Latin America [1]. Histoplasmosis is hard to diagnose because it requires mycological expertise and because fungal growth is slow. Moreover, histoplasmosis is frequently associated with tuberculosis (ranging from 2 to $38 \%$ and in most

\footnotetext{
*Correspondence: mathieu.nacher66@gmail.com

${ }^{1}$ Centre d'Investigation Clinique, CIC INSERM 1424, Centre Hospitalier de Cayenne, 97300 Cayenne, French Guiana

Full list of author information is available at the end of the article
}

countries $>10 \%$ ) [2]. This diagnostic challenge is estimated to cause large numbers of deaths often attributed to tuberculosis [3-5]. It has been shown in Latin America that culture negative "tuberculosis" was associated with a 79\% hazard increase [6]. The interpretation of this finding was that perhaps patients with culture-negative "tuberculosis" in fact had something else than tuberculosis. In French Guiana, the diagnosis of histoplasmosis relies on direct examination or culture, and clinicians are very aware of its presence, look for it aggressively and treat it presumptively whenever they suspect it, but they do not have routine access to antigen detection. With an incidence of 9.5 per 1000 person-years, tuberculosis is the 4th most frequent opportunistic infection among patients with HIV in French Guiana [7, 8]. Awareness 
of the presence of histoplasmosis started in the 1980s when the first cases of disseminated histoplasmosis with cutaneous and/or mucous lesions were diagnosed by dermatologists [9]. In the 1990s, following the introduction of fungal culture, the number of diagnoses greatly increased, and the number of deaths declined [10]. In a previous study we showed that disseminated histoplasmosis was the most frequent AIDS-defining infection in French Guiana [8]. In the present study, our objective was to identify historical risk factors for death, taking into account the immunological status, the main opportunistic infections [8], and their respective treatment.

\section{Main text Methods}

We studied French Guiana's HIV cohort data between 1992 and 2008 to search for factors associated with death in a cohort 2323 patients. HIV-infected persons in French Guiana receive free care and treatment. Foreigners also receive free care and treatment and residence permits for treatment. All antiretrovirals, genotyping, viral load, all diagnostic tests are available in French Guiana. For histoplasmosis, diagnosis rests on direct examination, fungal culture and histopathology [11]; for tuberculosis, Pasteur institute performs examination and culture, and molecular diagnosis. Details about this cohort have been presented elsewhere [12]. Trained research technicians routinely collect demographic, clinical and biological data from patient records in the 3 hospitals of French Guiana. Opportunistic infections, treatment of opportunistic infections, deaths, antiretroviral treatments, CD4 and CD8 counts are collected. Survival analysis was performed with death as the failure variable. The selection of variables was based on the use of background knowledge method [13]. The pertinent variables selected were the level of immunosuppression, antiretroviral treatment, the time period (because different time periods correspond to differences in the diagnostic and therapeutic arsenal), and the main opportunistic infections in French Guiana [8]. Crude incidence rates were obtained for different variable modalities. In this retrospective cohort, we used Cox proportional hazards modeling. Adjustments were made for categorized initial CD4 counts $(<200$ CD4, 200-499, and $>499$ CD4 per mm3), CD8 counts $(<643$ (first quartile), $>=643 \mathrm{CD} 8$ per $\mathrm{mm} 3$ ), age, time period (1992-1995 (pre-Highly Active Antiretroviral Treatment (HAART) and pre-fungal culture), 1996-2002 (postHAART and post-fungal culture and pre-ambisome), and 2003-2008 (post-ambisome)), ARV treatment, confirmed histoplasmosis, histoplasmosis treatment (deoxycholate/ liposomal amphotericin B and/or itraconazole), confirmed tuberculosis, tuberculosis treatment. Interaction terms were generated for each opportunistic infection and its treatment. Data were analyzed with STATA@ 15.

\section{Results}

Overall 2323 patients (1234 women and 1089 men) contributing 40,443 records were followed for a total 9608 person-years. The median follow-up duration was 2.9 years. The main nationalities were: French 499 (23.7\%), Haitian 903 (42.8\%), Guyanese 218 (10.3\%), Surinamese 246 (11.7\%), and Brazilian 167 (7.9\%). The median age at censoring was 39 years $(25 \%-75 \%$ quartiles $=31.07-48.07$ years). At censoring the median CD4 count was 311 per $\mathrm{mm} 3$ (25\%-75\% quartiles $=121-497$ $\mathrm{CD} 4 / \mathrm{mm} 3$ ) and the median CD8 count was 849 per mm3 $(25 \%-75 \%$ quartiles $=543-1238 / \mathrm{mm} 3)$. At the time of the last observation 1251 persons were on antiretroviral treatment (53.8\%).

There were a total of 370 deaths ( 3.85 deaths/100 person-years). The median time to death was 2.3 years $(25 \%$ $75 \%$ quartiles $=0.8-4.5$ years). The incidence rate of death varied between time periods: 14.6 deaths/100 personyears before 1997, 4.4 deaths/100 person-years for 19972002 , and 1.5 deaths/100 person-years for 2003-2008. The model results are shown in Table 1 . Being on tuberculosis treatment was associated with a greater hazard of death, adjusted Hazard Ratio $(\mathrm{aHR})=2.44$ (95\% CI 1.65-3.60)) . The diagnoses of confirmed tuberculosis $(\mathrm{aHR}=5.37$ (95\%CI 2.2912.58)), of histoplasmosis $\quad(\mathrm{aHR}=1.97$ (95\%CI 1.19-3.26)), of toxoplasmosis $(\mathrm{aHR}=3.52(95 \% \mathrm{CI}$ 1.97-6.28)), and pneumocystosis $(\mathrm{aHR}=4.18 \quad(95 \% \mathrm{CI}$ $1.48-11.80)$ ) were significantly and independently associated with death. Esophageal candidiasis, one of the most frequent opportunistic infections, was not independently associated with death and removed from the model. When looking at interactions between cotrimoxazole treatment and pneumocystosis, or between confirmed tuberculosis and tuberculosis treatment, there was a significant protective effect. It is of note that all patients who received anti-tuberculosis treatment $(n=347)$ did not have a final diagnosis of tuberculosis $(\mathrm{n}=93)$. For histoplasmosis, 199 patients received antifungal treatment while 141 were diagnosed as having histoplasmosis. The Cox model showed that the main opportunistic infections (except esophageal candidiasis, removed from final model) were independently associated with increased mortality (Table 1).

\section{Discussion}

The main opportunistic infections were associated with an increased risk of death after adjusting for CD4 count, age, period, and antiretroviral treatment. The interaction terms between treatment of tuberculosis and confirmed tuberculosis, and between treatment of pneumocystosis 
Table 1 Factors associated with death in a cohort of HIV-infected patients in French Guiana 19922008: multivariate Cox modeling

\begin{tabular}{|c|c|c|c|c|c|}
\hline & $\begin{array}{l}\text { Time at risk } \\
\text { (person-years) }\end{array}$ & Incidence rate & $\begin{array}{l}\text { Adjusted } \\
\text { hazard ratio }\end{array}$ & $\begin{array}{l}95 \% \\
\text { confidence } \\
\text { interval }\end{array}$ & $\mathbf{P}$ \\
\hline Age $<30$ years & 1797 & 2.28 & 1 & & \\
\hline Age $[30-40$ years] & 2998 & 4.16 & 1.69 & $1.10-2.59$ & 0.01 \\
\hline Age $[40-60$ years $]$ & 4116 & 3.76 & 1.47 & $1.14-2.64$ & 0.009 \\
\hline Age $>60$ & 696 & 7 & 6.16 & $3.70-10.27$ & 0.000 \\
\hline 1991-1996 time-period & 773 & 14.6 & 5.29 & $3.56-7.87$ & 0.000 \\
\hline 1997-2002 time-period & 4182 & 4.47 & 2.68 & $1.96-3.68$ & 0.000 \\
\hline 2003-2008 time-period & 4652 & 1.5 & 1 & & \\
\hline CD8 < first quartile $\left(643 / \mathrm{mm}^{3}\right)$ & 2156 & 7.2 & 1.77 & $1.37-2.28$ & 0.000 \\
\hline $\mathrm{CD} 8>=643$ per $\mathrm{mm} 3$ & 6716 & 2.09 & & & \\
\hline CD4 $<50$ & 645 & 23.25 & 36.02 & $15.41-84.20$ & 0.000 \\
\hline CD4 [50-200] & 1809 & 5.14 & 12.34 & $5.32-28.63$ & 0.000 \\
\hline CD4 [200-500] & 4387 & 1.18 & 4.33 & $1.86-10.11$ & 0.001 \\
\hline$C D 4>500$ & 2167 & 0.27 & 1 & & \\
\hline Antiretroviral treatment & 4938 & 1.47 & .30 & $0.22-0.40$ & 0.000 \\
\hline No antiretroviral treatment & 4670 & 6.35 & & & \\
\hline Tuberculosis (any location) & 62 & 36.69 & 5.37 & $2.29-12.58$ & 0.000 \\
\hline No tuberculosis & 9546 & 3.63 & & & \\
\hline Tuberculosis treatment & 238 & 24.3 & 2.44 & $1.65-3.60$ & 0.000 \\
\hline No tuberculosis treatment & 9370 & 3.32 & & & \\
\hline Interaction confirmed tuberculosis and tuberculosis treatment & & & 0.20 & $0.07-0.60$ & 0.004 \\
\hline Histoplasmosis & 105 & 35.89 & 1.97 & $1.19-3.26$ & 0.008 \\
\hline No histoplasmosis & 9502 & 3.49 & & & \\
\hline Histoplasmosis treatment & 289 & 7.2 & .79 & $0.37-1.57$ & 0.50 \\
\hline No histoplasmosis treatment & 9319 & 3.74 & & & \\
\hline Interaction confirmed histoplasmosis and antifungal treatment & & & 1.09 & $0.37-3.15$ & 0.86 \\
\hline Pneumocystosis & 70 & 17.06 & 4.18 & $1.48-11.80$ & 0.007 \\
\hline No pneumocystosis & 9538 & 3.75 & & & \\
\hline Cotrimoxazole & 2230 & 6.77 & 1.44 & $1.08-193$ & 0.01 \\
\hline No cotrimoxazole & 7378 & 2.96 & & & \\
\hline Interaction pneumocystosis cotrimoxazole & & & 0.13 & $0.03-0.49$ & 0.003 \\
\hline Toxoplasmosis & 86 & 44.14 & 3.52 & $1.97-6.28$ & 0.000 \\
\hline No toxoplasmosis & 9522 & 3.48 & & & \\
\hline
\end{tabular}

and confirmed pneumocystosis were logically associated with a decreased risk of dying. Cotrimoxazole treatment was independently associated with greater mortality, possibly because it reflected prophylaxis in advanced-HIV, but also because it reflected presumptive treatment. Tuberculosis treatment by itself was independently associated with an increased risk of death. Toxicities and drug-drug interactions may eventually lead to fatal outcomes. Nonetheless, another more plausible interpretation is that, in cases where the diagnosis is difficult, anti-tuberculosis treatment is given but that patients actually die from whatever disease caused TBlike symptoms.
Histoplasmosis is well known in French Guiana [14], nevertheless we may still be only seeing the upper part of the "iceberg". The present results were adjusted for 3 time periods of increasing awareness and diagnostic capacity, which suggests that whatever the time period, patients on anti-tuberculosis treatment were at greater risk of dying. Despite our promptness to think about it, despite our fungal culture and pathology lab, we still may not have fully realized how common histoplasmosis is. Had we used antigen detection, we would have found even more cases and avoided unnecessary tuberculosis treatments thereby preventing more deaths. This humbling realization suggests that scaling up the 
availability of histoplasma antigen detection tests and lipoarabinomannan (LF-LAM) antigen tests is important for the care of patients with advanced HIV disease, even in a resource-rich country. More generally, although WHO has screening guidelines for advanced HIV, French HIV recommendations no longer cover the diagnosis of opportunistic infections in advanced HIV $[15,16]$. However, patients are still often tested late with opportunistic infections. The evolution of the diagnostic arsenal and diagnostic and screening strategies should remain in HIV recommendations in order to obtain accurate diagnoses as early as possible and avoid fatalities due to undiagnosed pathogens.

\section{Limitations}

In 2009 the data collection system changed and we were not able to get the information after that. In the decade since then, as the cascade of care improved, mortality gradually declined in HIV-infected patients but the context of death remains similar $[17,18]$. Although antituberculosis treatment is specific, antifungal treatment is not specific to histoplasmosis, which makes it more difficult to ensure it was prescribed for histoplasmosis. Despite these limitations, this hospital cohort data brings an interesting light to the challenges faced by clinicians and shows gaps in the HIV recommendations. Finally, the data analysis could not test some of the hypotheses regarding the observed associations with death.

\section{Abbreviations}

aHR: Adjusted hazard ratio; AIDS: Acquired Immuno Deficiency Syndrome; CD4: Cluster of differentiation 4; CD8: Cluster of differentiation 8; HAART : Highly active antiretroviral treatment; HIV: Human Immunodeficiency Virus; LF-LAM: Lateral flow lipoarabinomannan; TB: Tuberculosis; WHO: World Health Organization.

\section{Acknowledgements}

We thank the clinical research technicians Ketty Bienvenu, Lidia Saint Louis, Sergine Soyon, and Karine Verin.

\section{Authors' contributions}

MN conceived the study, analyzed the data, and wrote the first draft; AA, PA, FD, MD, BG, E, PC included patients, acquired data, and critically reviewed the analysis and the manuscript. All authors read and approved the final manuscript.

\section{Funding}

None declared.

\section{Availability of data and materials}

The anonymized data may be made available but this would require permission from the Commission Nationale Informatique et Libertés (CNIL), 3 Place de Fontenoy, 75007 Paris, France.

\section{Ethics approval and consent to participate}

Patients included in the FHDH give written informed consent for the use of their data for research and publication of research results. Their identity is encrypted before sending the data to the Institut National de la Recherche Médicale (INSERM), which centralizes data from Regional Coordination for the fight against HIV (COREVIH) throughout France. This cohort was approved by the Commission Nationale Informatique et Libertés (CNIL) since Nov 27th 1991 and has led to numerous international scientific publications.

\section{Consent for publication}

Not Applicable.

\section{Competing interests}

No competing interests declared.

\section{Author details}

${ }^{1}$ Centre d'Investigation Clinique, CIC INSERM 1424, Centre Hospitalier de Cayenne, 97300 Cayenne, French Guiana. ${ }^{2}$ Département de Formation et de Recherche Santé (DFR Santé), Université de Guyane, 97300 Cayenne, French Guiana. ${ }^{3}$ Department of Infectious and Tropical Diseases, Centre Hospitalier de Cayenne, 97300 Cayenne, French Guiana. ${ }^{4}$ UMR Tropical Biome and Immuno-pathophysiology, Université de Guyane, 97300 Cayenne, French Guiana. ${ }^{5}$ Parasitology and Mycology Laboratory, Centre Hospitalier de Cayenne, 97300 Cayenne, French Guiana. ${ }^{6}$ Department of Dermatology, Centre Hospitalier de Cayenne, 97300 Cayenne, French Guiana.

Received: 26 February 2020 Accepted: 31 March 2020

Published online: 10 April 2020

\section{References}

1. Nacher M, Blanchet D, Bongomin F, Chakrabarti A, Couppié P, Demar M, et al. Histoplasma capsulatum antigen detection tests as an essential diagnostic tool for patients with advanced HIV disease in low and middle income countries: a systematic review of diagnostic accuracy studies. PLoS Negl Trop Dis. 2018;12:e0006802. https://doi.org/10.1371/journ al.pntd.0006802.

2. Caceres $\mathrm{DH}$, Valdes A. Histoplasmosis and tuberculosis co-occurrence in people with advanced HIV. J Fungi. 2019;5:73. https://doi.org/10.3390/ jof5030073.

3. Nacher M, Adenis A, Mc Donald S, Gomes MD, Singh S, Lopes IL, et al. Disseminated histoplasmosis in HIV-infected patients in South America: a neglected killer continues on its rampage. PLoS Negl Trop Dis. 2013;7:e2319. https://doi.org/10.1371/journal.pntd.0002319.

4. Adenis A, Nacher M, Hanf M, Basurko C, Dufour J, Huber F, et al. Tuberculosis and histoplasmosis among human immunodeficiency virus-infected patients: a comparative study. Am J Trop Med Hyg. 2014;90:216-23. https ://doi.org/10.4269/ajtmh.13-0084.

5. Adenis AA, Valdes A, Cropet C, McCotter OZ, Derado G, Couppie P, et al. Burden of HIV-associated histoplasmosis compared with tuberculosis in Latin America: a modelling study. Lancet Infect Dis. 2018;18:1150-9. https ://doi.org/10.1016/S1473-3099(18)30354-2.

6. Sterling T. Culture negative TB is associated with increased mortality in HIV-infected persons. 2015.

7. Guernier V, Guégan J-F, Deparis X. An evaluation of the actual incidence of tuberculosis in French Guiana using a capture-recapture model. Microbes Infect. 2006;8:721-7. https://doi.org/10.1016/j.micin f.2005.09.009

8. Nacher M, Adenis A, Adriouch L, Dufour J, Papot E, Hanf M, et al. What is AIDS in the Amazon and the Guianas? Establishing the burden of disseminated histoplasmosis. Am J Trop Med Hyg. 2011;84:239-40. https:// doi.org/10.4269/ajtmh.2011.10-0251.

9. Nacher M, Leitao TS, Gómez BL, Couppié P, Adenis A, Damasceno L, et al. The fight against HIV-associated disseminated histoplasmosis in the Americas: unfolding the different stories of four centers. J Fungi. 2019;5:51. https://doi.org/10.3390/jof5020051.

10. Adenis A, Nacher M, Hanf M, Vantilcke V, Boukhari R, Blachet D, et al. HIV-associated histoplasmosis early mortality and incidence trends: from neglect to priority. PLoS Negl Trop Dis. 2014;8:e3100. https://doi. org/10.1371/journal.pntd.0003100.

11. Huber F, Nacher M, Aznar C, Pierre-Demar M, El Guedj M, Vaz T, et al. AIDS-related Histoplasma capsulatum var. capsulatum infection: 25 years experience of French Guiana: AIDS. 2008;22: 1047-1053. https://doi. org/10.1097/qad.0b013e3282ffde67. 
12. Nacher M, Adenis A, Blanchet D, Vantilcke V, Demar M, Basurko C, et al. Risk factors for disseminated histoplasmosis in a cohort of HIV-infected patients in French Guiana. PLoS Negl Trop Dis. 2014;8:e2638. https://doi org/10.1371/journal.pntd.0002638.

13. Heinze $G$, Wallisch C, Dunkler D. Variable selection-a review and recommendations for the practicing statistician. Biom J. 2018;60:431-49. https ://doi.org/10.1002/bimj.201700067.

14. Vantilcke V, Boukhari R, Jolivet A, Vautrin C, Misslin C, Adenis A, et al. Fever in hospitalized HIV-infected patients in Western French Guiana: first think histoplasmosis. Int J STD AIDS. 2014;25:656-61. https://doi. org/10.1177/0956462413516299.

15. WHO| Guidelines for managing advanced HIV disease and rapid initiation of antiretroviral therapy. In: WHO [Internet]. [cited 15 Oct 2019]. http:// www.who.int/hiv/pub/guidelines/advanced-HIV-disease/en/.

16. Bressy J. Prise en charge du VIH - Recommandations du groupe d'experts. In: Conseil national du sida et des hépatites virales [Internet]. 6 Aug 2019 [cited 14 Nov 2019]. https://cns.sante.fr/actualites/prise-en-charge-duvih-recommandations-du-groupe-dexperts/.

17. Nacher M, Adriouch L, Huber F, Vantilcke V, Djossou F, Elenga N, et al. Modeling of the HIV epidemic and continuum of care in French Guiana. PLoS ONE. 2018;13:e0197990. https://doi.org/10.1371/journal.pone.01979 90

18. Article - Bulletin épidémiologique hebdomadaire. http://beh.santepubli quefrance.fr/beh/2020/2-3/2020_2-3_2.html.

\section{Publisher's Note}

Springer Nature remains neutral with regard to jurisdictional claims in published maps and institutional affiliations.
Ready to submit your research? Choose BMC and benefit from:

- fast, convenient online submission

- thorough peer review by experienced researchers in your field

- rapid publication on acceptance

- support for research data, including large and complex data types

- gold Open Access which fosters wider collaboration and increased citations

- maximum visibility for your research: over $100 \mathrm{M}$ website views per year

At BMC, research is always in progress.

Learn more biomedcentral.com/submissions 